\section{Abstractions}

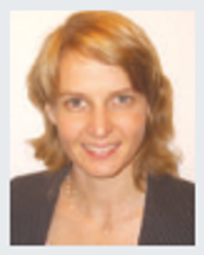

\section{FIRST AUTHOR}

Papua New Guinea offers scientists studying social interactions something of an island 'laboratory'. It is hometo many small indigenous groups with strong, cohesiveidentities and distinct cultural and social norms. In addition, their social systems of justice are less centralized than those of modern Western society. Helen Bernhard and her colleagues from the Institute for Empirical Research in Economics at the University of Zurich, Switzerland, went there to test how judgement of altruistic behaviour, such as fair sharing of goods, depends on group affiliation.

The scientists refereed aseries of thirdparty punishment games involving 195 people from two indigenous groups. Games comprised three players. One shared a given amount of money with a second, and the third judged the fairness of the transaction and had the chance to'punish' the first player for unfair behaviour. The aim was to find out whether the 'judge' favoured members of his or her owngroup when deciding the fairness of others' behaviour. And group affiliation did have an important role - judges favoured members of their own group, even supporting unfair behaviour (see page 912). Nature caughtup with Bernard to discuss this work.

What was it like working with the New Guineans?

They were eager to take part and enjoyed the games, which was very motivating. But after having explained the samegame and the same examples more than 200 times, I was also happy when the experiments were over.

Did you feet the New Guineans were studying you as well?

I am suremy presence and the games provided an interesting topic for conversation. But because I grew up in Papua New Guinea and lived there for 16 years, I probably didn't behave like a complete foreigner.

Switzerland is multiethnic. Did this have any influence on your choice of research? Yes. Given the omnipresence of groups in our modern societies we felt it was necess ary to understand the effects of group affiliation on human behaviour. Neglecting this would have left an important and interesting feature of human behaviour unexplained.

Is this work relevant to modem societies? The New Guinean clans are a special subject pool, so we must be careful about generalizing our results. However, a subsequent study of altruistic punishment in the Swiss army replicated the result that the willingness to punish is much higher if the victim of the violation belongs to the punisher's group. This indicates that our results might also be applicable to modern societies.

\section{MAKING THE PAPER}

\author{
Yigal Meir
}

\section{Electrons flow towards smaller, faster computers.}

Yigal Meir wanted a puzzle to solve during a sabbatical at Princeton University in New Jersey. Meir, a theoretical physicist at Ben Gurion University in Israel, and his host, Ned Wingreen, decided to tackle an anomaly that has baffled condensed-matter physicists for over a decade.

When researchers first studied the way electrons flow through a quantum-point contact, a small constriction connecting large reservoirs, they expected the rate to increase as the gap grew bigger, in steps of a universal value. The rate did, but with an additional first step of about 0.7 times the expected universal value. At first, this ' 0.7 anomaly' was thought to arise from irregularities in the device. But similar effects were found in almost all experiments involving quantum-point contacts, hinting that something in the quantum gap's environment influences electron flow. This anomaly has been problematic for researchers trying to use quantum mechanics to make smaller, faster computing devices, because such devices demand that no outside environmental factors affect the circuits.

Meir and Wingreen began by studying the available data. They noticed that the anomaly emerged at low temperatures, and that when the temperature was further reduced the conductance began to rise from 0.7 to 1 . "This looked very similar to the Kondo effect," says Meir. "This occurs during the process of electron transport through 'quantum dots' - small spaces to which electrons are confined with the help of several quantum-point contacts."

Meir and Wingreen asked Charles Marcus, an experimentalist at Harvard University, to look for a signature of the Kondo effect in quantumpoint contacts at low temperatures. Marcus confirmed their hunch, but this raised another issue. The Kondo effect describes electron scattering by a magnetic impurity and requires at least one

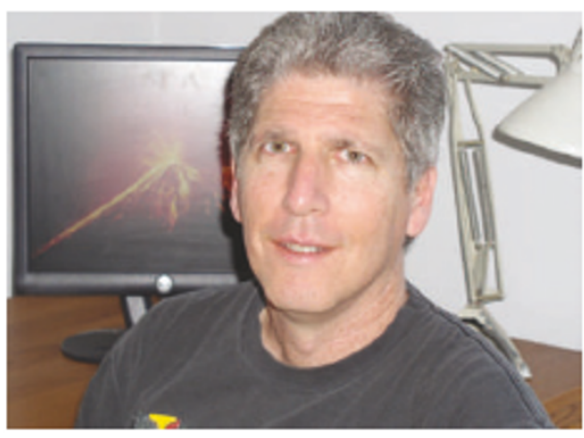

confined electron. Electrons in quantum-point contacts seem to be free from such constraints.

Despite this, Meir and Wingreen decided to invoke the existence of a magnetic impurity in a quantum-point contact to see if that would explain the anomaly. They assumed a magnetic moment in their theoretical calculations that explained the 0.7 anomaly, including its temperature and magnetic-field dependence.

But they needed to identify the proposed impurity to overcome "scepticism from the physics community, which centred on the puzzle of how a magnetic moment could form in such a system, Meir says. A dassical analogy of a quantum-point contact is a sea of electrons around a hill, ' The existence of a magnetic impurity on the point contact is equivalent to the formation of a puddle of water at the top of the hill - a counterintuitive phenomenon," says Meir.

"To find out more, I asked my postdoc, Tomaz Rejec, to develop a spin-density-functional theory calculation that could describe the experimental system," Meir says. Rejec found that the interplay of spin, electronic repulsion and quantum effects caused a small dip near the top of the hill' where an electron and its spin can be captured, forming a magnetic impurity.

This is both good and bad news for quantum computer devices based on quantum dots, says Meir. Magnetic impurities at point contacts would render such computers inoperable. But the magnetic impurity is formed only when conductance through the point contact is around 0.7 , so setting the conductance of each contact below that value should allow a circuit formed by quantum dots to function.

\title{
KEY COLLABORATION
}

More than thirty years ago, Kari Eskola and his colleagues suggested the existence of a relatively stable, excited-state isomer of the heavy element nobelium. Decades later, Eskola was part of a research team that carefully characterized the isomer in an effort to determine the existence of elements heavier than those listed in the current periodic table.

The original proposal was made by Eskola and others working at the Lawrence
Berkeley National Laboratory in

California on the basis of signs of radioactive decay - forensic clues that the isomer existed. But they couldn't be sure. Now, groups from the University of Liverpool, UK, and the University of Jyvăskylă in Finland - armed with much betterdetectors - have led research examining the isomer directly. "The biggest challenge was upgrading all aspects of the experimental apparatus," says lead author Rolf-Dietmar
Herzberg, a physicist at the University of Liverpool.

Their work bolsters the argument for the 'island of stability", a theoretical group of potentially stable elements that are much heavier than any currently noted on the periodic table (see page 896).

Although his role in the new work was minor, Eskola, now at the University of Helsinki, says he was glad to provide a "connecting link" with the original research. 